\title{
TradAction: un proyecto colaborativo de Aprendizaje Servicio gestionado con Padlet
}

\section{TradAction: a Service-learning project managed by Padlet}

\author{
AinhoA CusÁcovich \\ Universidad de Valladolid \\ ainhoa.cusacovich@uva.es
}

\begin{abstract}
The current situation of the globalized society makes us consider new educational paradigms, a necessary innovation in the processes, instruments and objectives of education. That latter point is the basis of the theories on LearningService, the "what for" approach, finding an answer in initiatives that improve the social environment close to the student. The application of curricular knowledge to solve social problems is a great motivation for students, teachers and partners of the TradAction project of solidarity translation. It combines, as we will analyse in this article, all the characteristics of hybrid learning: asynchronous and synchronous, formal and informal, individual and collaborative, theoretical and practical. Padlet is proposed, due to its exchange possibilities, as a management tool for this project based on cooperation
\end{abstract}

Key words

hybrid learning, ICT, collaborative, LearningService, Padlet.

\section{Résumé}

Les circonstances actuelles de la société mondialisée nous font envisager de nouveaux paradigmes éducatifs, une innovation nécessaire dans les processus, les instruments et les objectifs de l'éducation. Ce dernier point est la base des théories sur l'Apprentissage par le Service (ApS), l'approche du "pour quoi faire", qui trouve sa réponse dans des initiatives qui améliorent l'environnement social, plus ou moins proche de l'étudiant. L'utilisation des connaissances académiques pour résoudre des problèmes sociaux est une grande motivation pour les étudiants, les enseignants et les partenaires du projet TradAction de traduction solidaire. Il combine, comme nous l'analyserons dans cet article, tous les éléments présents dans l'apprentissage hybride: asynchrone et synchrone, formel et informel, individuel et collaboratif, théorique et pratique. Padlet est proposé, en raison de ses possibilités d'échange, comme outil de gestion de ce projet basé sur la coopération.

\section{Mots-clés}

apprentissage hybride, TIC, collaboratif, Apprentissage par le Service, Padlet. 


\section{Introducción}

\subsection{El ApS y sus características}

El Aprendizaje-Servicio (ApS) es una pedagogía que consiste en generar beneficios a la comunidad que nos rodea utilizando las competencias y contenidos a desarrollar en un determinado currículo. Es decir, se trata de un intercambio en el que, tanto el alumno como el entorno, se benefician de una actividad de aprendizaje.

La definición del concepto "Aprendizaje-Servicio" es compleja, pues abarca una serie de conceptos abstractos que comprenden la "reciprocidad", la responsabilidad, el "aprendizaje experiencial" (Deeley, 2016: 24), o la reflexión sobre el propio aprendizaje y su alcance en la sociedad. De esta forma, "las características clave del aprendizaje-servicio son el aprendizaje y el compromiso cívico del alumno. Unidos, estos aspectos vinculan la academia con la comunidad" (ibid.: 25).

La reflexión sobre el aprendizaje, que debe estar cuidadosamente guiada, supone la clave de esta metodología, pues sirve de puente entre la teoría y la práctica, entre el mundo virtual del aula y el real, presente en la sociedad. Recapacitar sobre el proceso de aprendizaje, la utilidad y el alcance de lo estudiado para mejorar el entorno, constituye uno de los mayores potenciales del método. Se trata, en definitiva, de una conciencia social que debe partir también del entorno educativo, también en el ámbito universitario. Aunque pueda resultar un planteamiento algo utópico, resulta necesario un ejercicio de responsabilidad social en su carácter más ético:

Es decir, un modelo de universidad que, además de preocuparse por la calidad, orienta su modelo formativo y su actividad docente, investigadora y de transferencia del conocimiento al logro de más inclusión social, a la formación de titulados que actúen desde perspectivas orientadas al logro del bien común y de una sociedad más justa y democrática. (Martínez, 2008: 16)

La historia del Aprendizaje-Servicio como método tiene una larga trayectoria. No resulta, sin embargo, sencillo delimitar el comienzo o una fecha en la que surge. En Estados Unidos, la necesidad de fomentar el compromiso en la ciudadanía se remonta a varias décadas atrás (Deeley, 2016: 21). Esta cuestión se mantiene a lo largo de los años, con un claro reflejo en organizaciones como Voluntary Services Overseas, fundada en 1958, que ya en la época reconoce los beneficios de los servicios a la comunidad en el entorno educativo (ibid.). En Norteamérica, fue John F. Kennedy quien, en 1961, recogió ya en una ley la introducción de los Peace Corps. Esta segunda organización nace de la necesidad de cambiar el curriculum de la enseñanza universitaria en Estados Unidos, vapuleado por los efectos de la guerra de Vietnam y la intrusión de la política en la educación (ibid.: 22). En 1985 nace Campus Compact, un programa nacional estadounidense para promover, a través del voluntariado, el compromiso cívico de los alumnos en etapas de secundaria y universidad (ibid.). 
En las últimas décadas, han surgido prácticas educativas y asociaciones que comparten, trabajan y analizan esta metodología, entre las que destaca la Red Española de $A p S^{1}$ en nuestro país, la $R E-A S E^{2}$ en Chile o la $S A H E C E$ en Sudáfrica. Existen redes como la Red Iberoamericana de Aprendizaje-Servicio (REDIBAS) que une a organismos públicos, $O S C^{3}$ y universidades de Sudamérica, España y Estados Unidos. Existe ya, desde 2001, la IARSL$C E^{4}$ (Asociación Internacional de Investigadores de Aprendizaje y Servicio y Compromiso Comunitario) centrado en una proyección internacional de esta metodología, a nivel académico. Finalmente, la Universidad de Barcelona y CLAYSS ${ }^{5}$ (Centro Latinoamericano de Aprendizaje y Servicio Solidario) dirigen la primera revista académica en lengua castellana: RIDAS6 (Tapia, 2017: 63-64).

En definitiva, numerosas iniciativas han tenido como objetivo vincular lo académico con lo social, competencias y aprendizajes con compromiso y cooperación. De esta idea parte el proyecto TradAction, que surgió como Proyecto de Innovación Docente en 2019 en la Facultad de Filosofía y Letras de la Universidad de Valladolid. En él, se vinculan las competencias de varias titulaciones filológicas con las necesidades de traducción existentes en numerosas ONG. El resultado: alumnos motivados, que se sienten útiles socialmente, comprometidos y aplicando conceptos de su curriculum en situaciones que favorecen a otros.

Este artículo presenta una experiencia didáctica basada en la metodología de Aprendizaje-Servicio (ApS): el proyecto TradAction. Dicha experiencia se ha llevado a cabo en asignaturas de lengua francesa de diferentes grados de la Universidad de Valladolid.

Comenzaremos planteando los colaboradores y participantes del proyecto en un primer apartado, para continuar con las bases teóricas que cimentan esta metodología. En los siguientes apartados se analizará el carácter democrático del proceso de aprendizaje en este proyecto, así como el desarrollo de competencias y la transdisciplinaridad que sustentan el $A p S$. Este segundo apartado se completará con una valoración del proceso de evaluación y coevaluación en este entorno de aprendizaje dinámico.

Ya en el tercer apartado abordaremos la herramienta escogida para gestionar el ApS: Padlet. Además de sus funcionalidades, analizaremos en qué medida favorece el intercambio y la cooperación de los alumnos. Se revisará para ello el concepto de "Trabajo en red" y la reflexión como eje vertebrador de la metodología.

El cuarto apartado relacionará el proyecto TradAction con el concepto de aprendizaje

1 https://www.aprendizajeservicio.net/ [25-03-2021].

2 https://reasechile.wordpress.com/ [25-03-2021].

3 Las siglas OSC corresponden al término de organización de la sociedad civil. Se trata de un término más amplio que el de ONG, pues incluye cualquier organización formal o informal de la sociedad civil, incluyendo medios de comunicación, centros de investigación, empresas, ciudadanos individuales u organizaciones populares que persiguen un mismo objetivo común. Las OCS incluyen, por tanto, las propias ONG (Fuente: web de FAO: http://www.fao.org/tempref/GI/Reserved/FTP_FaoRlc/old/ong/ongosc.htm [23-03-2021].

4 https://www.researchslce.org/ [25-03-2021].

5 https://clayss.org.ar/redibero.html [25-03-2021].

6 https://revistes.ub.edu/index.php/RIDAS [25-03-2021]. 
híbrido, revisando las características de éste. Finalizaremos con unas conclusiones sobre la base teórica de la metodología y en qué medida resulta enriquecedor para todos los participantes este tipo de metodología.

\subsection{Las asignaturas implicadas en el proyecto y los colaboradores}

Utilizamos la metodología Aprendizaje-Servicio para tratar aspectos presentes en el curriculum de varias asignaturas, implicando y entrenando diversas competencias de todas ellas, utilizando los contenidos que componen sus temarios. Las asignaturas centrales de las que parte el proyecto son las de Francés como Lengua Moderna con Fines Académicos I y II, que se imparten como parte de la titulación del Grado de estudios hispánicos y el Grado de estudios clásicos de la Universidad de Valladolid.

Se trata, por tanto, de un alumnado con un perfil muy determinado, con formación filológica no muy avanzada, ya que se encuentran en primer curso de sus estudios, pero, por lo general, reflexivo con los procesos de traducción del español hacia otras lenguas y viceversa.

Contamos, además, con la colaboración de alumnado de cuarto del Grado de Lenguas Modernas de la facultad, cuya especialidad es francés. El profesorado de varias asignaturas también está implicado, con lo que las labores de corrección y revisión se aseguran, logrando una gran calidad en los productos que presentamos después a las ONG que participan en el proyecto.

Nuestros socios son ONG para las que realizamos tareas de traducción interna que no requieren traducción oficial. Hemos trabajado, así, con Permondo ${ }^{7}$, que, a su vez, gestiona traducciones de varias ONG que solicitan su ayuda. Actualmente, los encargos nos llegan de Eclosio $^{8}$, una ONG vinculada a la Universidad de Liège (Francia), que lleva a cabo numerosos proyectos por todo el mundo, relacionados con agroecología, agricultura sostenible, igualdad de género en el entorno campesino y productor, y alimentación saludable.

El ApS se basa en la combinación del aprendizaje basado en la experiencia y el compromiso social en el que se aplica ese aprendizaje. A través de este tipo de proyectos se desarrollan competencias personales e interpersonales, así como académicas y profesionales que ayudarán además al alumno a una mejor adaptación al entorno laboral. Se fomentan la responsabilidad personal, el pensamiento crítico y la reflexión, así como el compromiso social.

En cuanto a las fases en que se dividió el proyecto, primeramente, se creó con los alumnos anteriormente citados una "agencia de traducción" encargada de documentos internos de utilidad para la ONG en sus acciones de difusión con los socios españoles y sudamericanos. En esta primera fase, presentamos la ONG destinataria de las traducciones y dividimos los equipos de trabajo, centrando en cada uno labores diferenciadas de documentación, traducción, revisión y corrección de estilo. La segunda fase consistió en realizar la investigación sobre el tipo de texto y campos semánticos empleados en él. En la tercera fase

7 http://www.permondo.eu/es/ [25-03-2021].

8 https://www.eclosio.ong/ [25-03-2021]. 
los alumnos tradujeron los textos y los correctores de cuarto curso del grado llevaron a cabo las correcciones y revisiones.

Los objetivos del proyecto fueron los siguientes:

Objetivo 1: Tomar conciencia del trabajo de la ONG y de la importancia del conocimiento del francés para la mediación y la ayuda en la sociedad.

Objetivo 2: Comprender el funcionamiento de una agencia de traducción y las cualidades profesionales necesarias para trabajar en ella.

Objetivo 3: Adquirir las destrezas comunicativas y el vocabulario suficientes para llevar a cabo las traducciones.

En lo que respecta a la evaluación, a los alumnos se les entrega un cuestionario al final del proyecto para autoevaluar su implicación y evaluar lo que esta actividad ha supuesto para ellos. Se realiza también una coevaluación que llevan a cabo los alumnos correctores con los que han realizado las traducciones, y que permite al profesor tener un dato más de evaluación. Finalmente, la asociación rellena un cuestionario de valoración en el que evalúa si la acción de los alumnos ha sido realmente valiosa 9 .

El proyecto se está llevando a cabo con 15 alumnos en este momento, con intención de aumentar el número de alumnado implicado. En cuanto a sus valoraciones, el año pasado los alumnos manifestaron tener una mayor motivación al aplicar sus conocimientos en un entorno más real y sentir la utilidad de su aprendizaje. En lo referente a la herramienta Padlet, lo consideraron un buen canal de participación en el proyecto, por su flexibilidad en los horarios, la facilidad de uso y la posibilidad de colaborar con recursos propuestos por ellos mismos. Todos los alumnos a los que se les propuso mostraron un alto grado de motivación, pidiendo algunos participar este año de forma voluntaria en el proyecto. En cuanto a la adquisición de contenidos y competencias, declararon haber mantenido una actitud más reflexiva y un interés más profundo en lo lingüístico al trabajar con esta metodología.

\section{Un proceso enseñanza-aprendizaje más democrático}

A medida que el acceso a estudios universitarios se ha ido convirtiendo en un hecho más accesible, se ha visto la necesidad de acudir a la innovación educativa, en la intención de proporcionar la atención necesaria a un público cada vez más diverso. Esto nos lleva inevitablemente a una reflexión sobre "l'alignement pédagogique"10 (Poumay, 2014: 5), ese cambio necesario para incluir a todos los alumnos en el proceso. Esta cuestión pasa por motivarlos

9 Esta valoración no fue posible al finalizar el proyecto el curso pasado por circunstancias derivadas del confinamiento por Covid-19.

10 El concepto implica una alineación de objetivos, métodos y evaluaciones y es descrito del siguiente modo: "Il s'agit de mettre en cohérence les objectifs d'une formation (qui peuvent être des compétences à développer), les méthodes déployées pour y parvenir et la façon dont les apprentissages seront évalués. En résumé, pour favoriser la réussite de ses étudiants, l'enseignant organise l'apprentissage des notions qu'il a annoncé viser et 
para que puedan llegar a ser más dueños de su aprendizaje, explicar con claridad el objetivo de las tareas a desarrollar y permitirles que sean ellos mismos quienes las controlen, lo que nos llevará a tener estudiantes más activos.

Este aparente obstáculo se resuelve, al menos parcialmente, gracias a las nuevas tecnologías y la democratización del aprendizaje. El nuevo enfoque precisa un acento especial en las necesidades del alumno y una importancia cada vez mayor de las aportaciones y aprendizajes peer to peer, basadas en la colaboración entre iguales, para poder resolver determinados problemas o situaciones (Goastellec, 2014: 68).

Las redes de aprendizaje son, por definición, mecanismos más igualitarios, en los que el compromiso de participación y la colaboración se convierten en premisas. "La naturaleza de las tecnologías de redes tiende a democratizar la participación y a permitir una mayor interacción entre alumnos y entre los alumnos y sus profesores/ayudantes" (Harasim et al., 2000: 306).

La necesidad de centrarnos en el alumno y de otorgarle una mayor autonomía en la gestión de su aprendizaje, también implica cambios en nuestra labor como docentes.

[...] il s'agit notamment de détrôner l'enseignant comme barycentre du système d'enseignement universitaire pour y placer l'étudiant rebaptisé " apprenant ", cela en s'aidant des TIC pour penser des dispositifs pédagogiques visant à faire de l'apprenant un co-producteur de ses savoirs. (Valluy, 2012)

Esto no supone una tarea fácil, e implica tener en cuenta todos los canales de comunicación y la forma en que estamos “presentes” para los alumnos (Jézégou, 2014). Los lazos se convierten en algo esencial en el proceso de aprendizaje, sobre todo cuando se trata de la parte remota, haciéndose esencial un buen proceso de comunicación que acerque distancias.

Debemos, pues, revisar las tres dimensiones de vinculación entre docentes y alumnado: la presencia socio-cognitiva, la socio-afectiva y la pedagógica, que intervienen en todos los procesos de aprendizaje (Jézégou, 2014: 118). La primera se refiere a los intercambios llevados a cabo entre alumnos que tienen por objetivo cuestiones puramente curriculares. La presencia socio-afectiva implica todas las interacciones que se vinculan más con lo emocional o social. Es en el tercer tipo de presencia en el que los docentes entramos en juego, y donde nuestro papel puede tener un efecto de cohesión. Se trata de todas las labores de coordinación, dirección, gestión y moderación que se llevan a cabo con los estudiantes y que, a su vez, afectan a las otras dos presencias.

En cualquier caso, la comunicación supone uno de los ejes esenciales de la metodología $A p S$, en el que el avance de cada uno de los miembros se lleva a cabo precisamente desde la riqueza generada por sus intercambios.

dont il vérifiera la maîtrise. Il "aligne" ainsi les objectifs, les méthodes et les évaluations de son cours" (Poumay, 2014: 5). 
Una comunicación efectiva constituye, sin duda, la base de la metodología. La argumentación, el debate y el intercambio forman parte, de este modo, de un sistema que refuerza la reflexión y el espíritu crítico. La comunicación con los demás es vital para explorar diversas perspectivas, cuestionar ideas preconcebidas y compartir una red de apoyo. El resultado pueden ser unos pensadores independientes y críticos. El aprendizaje-servicio incorpora fácilmente estos factores dentro de un aula democrática. (Deeley, 2016: 47)

Sin embargo, la comunicación no es más que el eje vertebrador de los aprendizajes y contenidos. Como veremos, esta metodología supone un desarrollo de las competencias planteadas para cada área y favorece el intercambio entre materias y disciplinas, resultando una red de aprendizaje muy productiva.

\subsection{Desarrollo de competencias y transdisciplinaridad}

La participación del alumnado en proyectos $A p S$ implica, necesariamente, un enfoque competencial. Entendemos por competencia, en cualquier caso, la capacidad para "combinar la adquisición de recursos cognitivos y la disponibilidad para movilizarlos en contextos reales" (Martínez, 2008: 20).

No debemos confundir, sin embargo, un aprendizaje competencial, con las prácticas que los alumnos puedan llevar a cabo en laboratorios, hospitales o empresas, al finalizar sus estudios. Lo competencial tiene una gran carga teórica, enseñando, a su vez, a movilizar estos contenidos en entornos reales. Así, encontramos que un "modelo centrado en competencias requiere aprendizaje de contenidos procedimentales y actitudinales, pero también, y de manera especial, informativos y conceptuales" (ibid. 2008: 20).

El alumno debe ser capaz de emplear todas las estrategias y conocimientos de los que dispone por el bien común, y plantear soluciones a problemas que se puedan presentar en el desarrollo de las actividades. Se trata, por lo tanto, de la metodología que mejor conjuga teoría y práctica y que más claro muestra el potencial del aprendizaje que el alumno está llevando a cabo.

El aprendizaje-servicio permite proponer situaciones en las que deba haber comprensión, transferencia de conocimientos, resolución de problemas y reflexión crítica en un contexto real y complejo. Son situaciones en las que el alumno demostrará si es competente resolviendo la situación real propuesta. (Campo, 2017: 127)

En cualquier caso, para que un proyecto pueda considerarse como perteneciente a la metodología $A p S$, debiera integrar las siguientes características (Martínez, 2008: 24-25):

- Debe fomentar la actitud crítica y la reflexión moral de los estudiantes frente a cuestiones relevantes tanto social como éticamente.

- Debe estar basado en redes de intercambio que creen relaciones respetuosas en las que se reconozca el valor de cada una de las acciones de los componentes de la red. 
- Debe organizarse en base a tareas de carácter cooperativo y colaborativo, al mismo tiempo que se trabaja la reflexión individual sobre la tarea, con la utilización, por ejemplo, de porfolios.

- Debe suponer un aprendizaje práctico para la vida cívica en sociedad, formando en valores como "la libertad, la igualdad, la solidaridad, el respeto a uno mismo y a la naturaleza, la participación y la responsabilidad; valores que son exigibles por correspondencia con los valores de la justicia y la dignidad" (Martínez, 2008: 24).

- Debe favorecer la coevaluación entre iguales, así como la evaluación y valoración del trabajo por parte de todos los participantes en el proyecto.

Por último, el trabajo a través de esta metodología se caracteriza, en ocasiones, por un fuerte componente interdisciplinar. Dado el carácter real de las situaciones que se plantean, a menudo es necesaria la intervención de diferentes campos de conocimiento para dar solución a las problemáticas que se puedan plantear. Se trata, pues, de uno de los pilares de los proyectos $A p S$. La interdependencia y la cooperación constituyen la base y hacen avanzar el proyecto, que se enriquece con cada una de las aportaciones.

\subsection{Evaluación y coevaluación en un entorno de aprendizaje dinámico}

La evaluación constituye un punto esencial en cualquier método de enseñanza-aprendizaje, sus bases y justificación deben estar bien asentadas para que sea efectiva y útil para el alumno. Como bien sabemos, existen dos tipos de evaluación, una sumativa y otra formativa. La primera supone una visión del nivel de adquisición de los conocimientos y competencias. La segunda, sin embargo, implica un diagnóstico de la evolución y los posibles obstáculos con los que se está encontrando el alumno. Es en esta última en la que el feedback debe realizarse de manera exhaustiva e inmediata a cualquier evaluación, ya que si no, no tendría validez para impulsar el progreso del alumno y solventar los problemas encontrados.

Además, estos intercambios favorecen la metacognición en el alumno, quien, gracias a una reflexión crítica sobre su aprendizaje, puede ser consciente de sus logros y puntos débiles (Deeley, 2016: 143).

Uno de los tipos de evaluación más efectivos es, sin duda, la coevaluación, que consiste en delegar parte de la tarea evaluadora en los alumnos, estableciendo previamente criterios. Es muy útil, en este sentido, utilizar rúbricas comunes, con el fin de que la evaluación sea comprensible para los alumnos y potencialmente más objetiva. Aunque en ocasiones los docentes se puedan llegar a mostrar reticentes con este sistema, se trata de una herramienta que favorece un aprendizaje profundo. "La coevaluación implica compartir la responsabilidad del juicio con los alumnos; cosa que puede ser empoderadora e intrínsecamente motivadora para ellos". (ibid.: 147) 
El funcionamiento mediante trabajo colaborativo permite llevar a cabo una coevaluación por parte del alumnado en la que, en este contexto en particular, los alumnos francófonos evalúan las destrezas comunicativas y lingüísticas de sus compañeros, cuya lengua materna es el español, y viceversa. La coevaluación se efectúa, de esta forma, por parte de "expertos" que se encuentran ante problemas de traducción similares en su día a día, lo que permite a su vez una reflexión profunda sobre estructuras y mecanismos del lenguaje.

El alumno puede, además, tomar conciencia de sus errores en la traducción o en algún momento del proceso, ya que los textos se encuentran en continuo estado de revisión por parte del profesorado y de análisis por el resto de los compañeros. La posibilidad que ofrece Padlet de valorar los comentarios y recursos que se comparten permite que la autoevaluación surja casi de forma natural, al observar el feedback generado por los demás participantes en el proyecto.

La evaluación se produce, además, por parte del profesorado de la asignatura, así como de otros profesores del grado de Lenguas Modernas que valoran el proceso de traducción en su complejidad y las competencias necesarias para que el desarrollo y el resultado sean exitosos.

\section{Intercambio y cooperación a través de TIC: Padlet}

\subsection{Trabajo en red}

Uno de las principales “dinamismos pedagógicos” (Martín \& Puig, 2017: 23) que se plantean en esta metodología es la participación. Las acciones individuales de cada alumno repercuten en el proyecto colectivo, y su inacción conlleva consecuencias visibles a corto plazo. Esto empuja al alumnado a la participación activa, al generar un sentimiento de responsabilidad que no sería posible en un trabajo particular. El alumno firma, de este modo, un pacto que le otorga protagonismo y derechos, pero también unos deberes, que incluyen la aportación de propuestas, planificación de tareas, la gestión de las partes del proyecto y la toma de decisiones (ibid.). Esto transforma el proceso en algo tremendamente democrático, un entorno en el que la argumentación y el debate suponen parte de la práctica de lo competencial.

El trabajo en equipo se convierte, además, en un requisito indispensable, en el que el aprendizaje se produce en parte por la sinergia de sus componentes. Esto propone, entre otros beneficios, una mejora de las relaciones interpersonales y una mayor capacidad de diálogo y argumentación.

Ya existen investigaciones que han analizado el impacto del trabajo en red en los estudiantes y ellos han destacado numerosas ventajas a nivel de aprendizaje ${ }^{11}$ (Harasim, 1987).

11 Esta investigación fue desarrollada en un curso de posgrado en ciencias de la educación impartido en red. 
La primera está relacionada con una mayor interacción entre los alumnos, que se convierten a su vez en fuentes de aprendizaje. El aprendizaje colectivo se transforma en una realidad en la que cada componente aporta y es capaz de ayudar en algo. Destacan también la comodidad de acceso a los materiales, que pueden consultar a cualquier hora del día. Por último, los alumnos manifiestan un aumento en su motivación (Harasim et al., 2000: 49-50).

Se produce, de este modo, un acceso ampliado a la educación, que favorece, además, una reflexión más pausada y sin horarios:

Las redes anulan las restricciones temporales. La posibilidad de interacción asincrónica facilita la comunicación entre zonas horarias y aumenta el control del tiempo y del ritmo de la participación por parte del usuario. De esta manera el aprendizaje en red permite a los usuarios participar en el momento que les resulte más propicio y favorece una reflexión cuidadosa del proceso de interacción, tanto en el caso de los mensajes recibidos como los enviados. Las redes permiten vincular el hogar, el centro educativo y el lugar de trabajo. (Harasim et al., 2000: 302)

La metodología ApS plantea un entorno idóneo para la colaboración, centrándose en el trabajo en redes de aprendizaje, comprendiendo estas como "una estrategia de articulación e intercambio entre instituciones que deciden asociar voluntariamente sus esfuerzos, experiencias y conocimientos para objetivos comunes" (Campo, 2017: 129).

De esta forma, se cumple con uno de los principales objetivos de este tipo de metodología: el trabajo mediante la cooperación. Surge, entonces, una definición de las propuestas de aprendizaje-servicio en el entorno universitario, creada por Míquel Martínez:

[...] identificamos las propuestas aprendizaje servicio en la universidad como propuestas de trabajo cooperativo y/o colaborativo que, utilizando el lenguaje y los conocimientos de las disciplinas que conforman el plan docente de una titulación, desarrollan en el estudiante competencias orientadas a una mejor comprensión de la realidad social, económica, medioambiental, mediática, cultural y personal que afectan a los miembros de una comunidad, con voluntad de transformación social y contribuyendo a incrementar el bienestar de las personas y el nivel de inclusión social. (Martínez, 2008: 21)

\subsection{La reflexión como centro nuclear del aprendizaje}

La reflexión sobre el trabajo efectuado, el proceso y los mecanismos que se han desencadenado en la realización de las tareas se refleja, de manera sencilla, gracias a las herramientas como blogs o espacios colaborativos. Generamos, de este modo, un espacio de trabajo y reflexión que impide "caer en un activismo superficial” (Martín y Puig, 2017: 24). La reflexión constituye una parte nuclear de cualquier proyecto $A p S$, ya que permite un autoanálisis, así como una visión más objetiva de las posibles mejoras y cambios a realizar en el futuro en el método de trabajo o incluso, el objetivo último del proyecto. Se define, así, la reflexión como “continua, conectada, desafiante y contextualizada" (Eyler, Gides \& Schmiede, 1996: 16). 
Continua, ya que ocurre antes, durante y después de la experiencia; conectada, dado que es el vínculo entre el servicio y los intereses intelectuales y académicos del estudiantado; desafiante, al empujar a estudiantes a pensar en nuevas formas de resolver problemas, plantear nuevos retos e intereses, y contextualizada, es decir, apropiada para cada contexto. (ibid.)

La reflexión no se limita exclusivamente a una cuestión práctica en el aula para facilitar la comprensión de determinados conceptos. Va más allá, creando interconexiones con el resto de campos con los que el alumno interactúa en su día a día y supone un paso más en su capacidad de emitir juicios fundados. En la universidad, esta cuestión ya se ha planteado en términos de competencia a desarrollar. Los llamados "descriptores de Dublín" ${ }^{12}$, que establecen competencias y su obligado desarrollo en entornos universitarios, establecen, en su tercer apartado "[...] saber reunir e interpretar datos relevantes, normalmente dentro de su área de estudio, con el fin de emitir juicios que incluyan una reflexión sobre temas relevantes de tipo social, científico o ético" (Martínez, 2008: 22).

Estas reflexiones constituyen, además, un replanteamiento, en algunos casos, de la propia ciudadanía y el papel del individuo en la sociedad:

Las reflexiones críticas permiten que lo personal entre en el entorno académico formal. Esto requiere que la individualidad de cada alumno interprete o cuestione un conocimiento generalmente aceptado, las ideas preconcebidas y sus experiencias para construir sentido. La reflexión crítica es también la plataforma de lanzamiento para la praxis y la transferencia del pensamiento en acción. Para los alumnos de aprendizaje-servicio esto puede traer consigo un cambio personal, convirtiéndose en unos ciudadanos más activos en sus comunidades, así como tal vez instigar el cambio social y político. Las consecuencias potenciales son amplias, variadas y quizás ilimitadas. (Deeley, 2016: 101)

\subsection{Padlet como herramienta colaborativa}

Las nuevas tecnologías han supuesto un verdadero avance en lo referente a comunicación. Aunque el aprendizaje no se lleve a cabo de modo presencial, encontramos maneras de suplir esa "ausencia", de recurrir a redes que facilitan este trabajo de "aglomerado social" que constituyen los grupos de aprendizaje colaborativo:

Elles permettent ensuite une réduction temporelle, une immédiateté à la communication. Enfin, les technologies, par le réseautage social, multiplient la possibilité de créer et d'entretenir des relations sociales avec des acteurs de plus en plus nombreux. (Peraya, 2014: 28)

12 Se trata de una serie de descriptores utilizados como marco para determinar las cualificaciones en que se debe basar la educación superior. BOE: https://www.boe.es/buscar/pdf/2011/BOE-A-2011-13317-consolidado.pdf [25-03-2021]. 
A pesar de que la utilización de TIC en educación suponga, a menudo, una pérdida de comunicación en los momentos no presenciales de formación, en realidad nos encontramos ante nuevos tipos de comunicación, más plurales y adaptados a cada una de las realidades presentes en nuestros alumnos. El propio Peraya, analizando el modelo de "distance transactionnelle" de Jézégou (2007), establece los numerosos tipos de intercambio entre alumnos que suponen un enriquecimiento del aprendizaje. Todos estos tipos de interacción implican reflexión y negociación, es decir, suponen la adquisición de una serie de competencias difícilmente aprendidas en otros contextos.

Ce modèle s'appuie sur les conceptions de l'apprentissage social et du socioconstructivisme: les échanges, confrontations et négociations entre les membres, les retours critiques et le processus réflexif engendré par les échanges et les discussions favorisent le développement de connaissances individuelles tandis que la collaboration et l'expérience collective du groupe soutiennent un apprentissage de groupe en relation avec son propre fonctionnement, les mécanismes de régulation, l'adoption de principes et de méthodes scientifiques, le développement d'une dimension réflexive sur l'expérience vécue, etc. (Peraya, 2014: 30)

En los últimos años, han surgido numerosas herramientas colaborativas que facilitan la interacción y permiten a un número elevado de participantes intervenir en el proceso educativo. Las nuevas tecnologías han conseguido construir, organizar y gestionar el conocimiento y las vías en las que se transmite, facilitando la comunicación, el diálogo y la interacción online. De este modo, se pueden crear vínculos entre los diferentes agentes de un proyecto, independientemente de las distancias y organismos a los que pertenezca cada uno.

Padlet permite crear un entorno dinámico que facilita, en primer lugar, el debate y el intercambio de información entre todos los participantes en el proyecto.

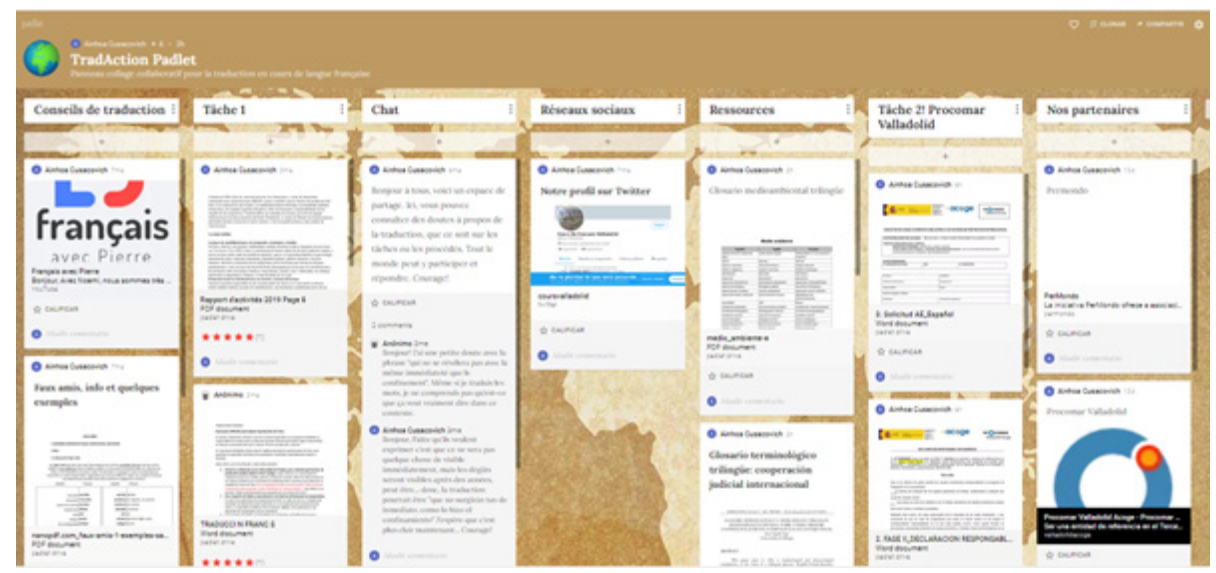

Figura 1. Tablón de Padlet del proyecto TradAction ${ }^{13}$.

13 Enlace del Padlet: https://padlet.com/ainhoacusacovich/zj9fv8uy232tyxdc [26/03/2021]. 
La herramienta Padlet consiste, básicamente, en un muro en el que todos los participantes del proyecto pueden compartir información, documentos, fotos, enlaces o cualquier material que consideren importante para la tarea a desarrollar. Una de las mayores ventajas es que la interacción se produce a tiempo real, por lo que se pueden establecer debates en torno a temas importantes o foros de dudas. La segunda ventaja es que se trata de una herramienta de fácil manejo, que no requiere crear cuentas ni perfiles, por lo que, además de acceder de forma rápida, no provocará incidencias con permisos. Se trata, además, de una web muy intuitiva, por lo que la barrera de lo digital no plantearía, en principio, ningún problema. Por último, dado su carácter eminentemente visual, se pueden crear fácilmente productos basados en Visual Thinking que pueden compartirse y enriquecer a todos los miembros del equipo, favoreciendo los procesos de aprendizaje de los alumnos.

Además, existe la posibilidad de dividir y asignar las tareas a los grupos que se estime necesario. Esto resulta especialmente interesante en proyectos como el que nos planteamos, pues la "agencia de traducción" en que convertimos el aula tenía, a su vez, un equipo de documentalistas, otro de traducción propiamente dicha y otro de corrección de estilo, formado por alumnos cuya lengua materna era la lengua destino.

Las aportaciones de cada participante del proyecto, tanto de profesores, como miembros del partenariado (ONG u otros) o alumnos, pueden ser anónimas o reflejar el nombre del participante. Resulta una buena opción, ya que, si lo que necesitamos es un brainstorming, quizá haya más participación si esta es anónima, mientras que las participaciones firmadas pueden servirnos para la evaluación.

\section{TradAction, un ejemplo de blended learning}

La definición de "aprendizaje híbrido" no es sencilla, y se ha ido modificando a lo largo de los años, sin embargo, podemos considerar blended learning la formación que "pourrait combiner une ou plusieurs des dimensions suivantes: en ligne/hors ligne, individuel/ collaboratif, contenu formel/informel, théorie/pratique" (Peraya et al., 2014: 17).

El proyecto TradAction conjuga, si no todos, la mayoría de estos aspectos, convirtiéndose así en un entorno educativo flexible con un gran potencial. En primer lugar, el proceso de enseñanza-aprendizaje se produce en las clases presenciales, en las que se intercambian aportaciones y comentarios; pero también de manera online, pues, tanto las conclusiones como cada una de las etapas del proceso son reflejadas en Padlet por el alumno y los demás colaboradores, de forma asíncrona.

Por otra parte, y como ya hemos señalado, se trata de un aprendizaje individual, que incluye trabajo y reflexión personal, pero también una colaboración estrecha con cada uno de los componentes del proyecto, buscando un producto, la traducción en este caso, que se va modificando y mejorando con ayuda de la cooperación de todos los agentes. Debemos añadir 
que algunos materiales del Padlet, como los recursos y webs de estudio que se comparten, forman parte de un contenido formal, sin embargo, también se producen intercambios de contenido informal en el foro de dudas y en los recursos que los alumnos pueden compartir libremente con los demás miembros del equipo.

Por último, la combinación entre teoría y práctica del proyecto TradAction es evidente. Existen recursos en los que se expone el proceso de traducción, así como algunos en los que se explica los diferentes problemas con los que podemos encontrarnos. Sin embargo, se trata de tareas eminentemente prácticas, en las que el producto generado es, además del resultado de la reflexión y la colaboración entre los agentes, un bien en sí, susceptible de mejorar el bienestar de otras personas en varias partes del mundo.

Pertenecería este proyecto, entonces, con la ayuda de Padlet, a lo que Peraya llama "dispositifs hybrides", en este caso, trabajados a distancia parcialmente, que permite las siguientes funciones pedagógicas:

[...] mise à disposition de ressources; activités de production, d'écriture individuelle ou collective; communication synchrone et asynchrone; soutien et accompagnement des étudiants; évaluation et auto-évaluation en ligne (QCM et Quiz); gestion des évaluations et des dossiers d'étudiants, etc. Ce ne sont donc plus les seuls contenus qui se trouvent médiatisés par de tels environnements technopédagogiques, mais bien le dispositif de formation dans toute sa complexité. (Peraya et al., 2014: 17)

En la formación presencial se explican los recursos que se utilizarán en línea, se debate sobre las posibles dificultades y se difunden los contenidos que normalmente habríamos aportado en el aula. De esta forma, la parte no presencial libera a la parte presencial de las tareas de transmisión de contenido y difusión, dejando más espacio para la interacción entre estudiantes y docente y viceversa (ibid.).

Debemos introducir, en este punto, el concepto de "concepciones del aprendizaje" (Marton et al., 1993), vinculado a la pregunta “¿Para qué estudio lo que estudio?”. La variación en alguna o varias de estas percepciones nos indicaría de algún modo, solo desde el punto de vista del estudiante, que se ha producido una mejora significativa en el proceso de enseñanza- aprendizaje en cuanto a motivación. Estas concepciones se clasifican del siguiente modo, siendo las tres primeras más básicas y las tres últimas más profundas: " 1 . une augmentation quantitative de connaissances; 2. mémoriser et reproduire; 3. appliquer; 4. comprendre; 5 . voir quelque chose de manière différente; 6 . changer comme personne" (ibid.). Resulta interesante destacar que en los procesos de aprendizaje basados en esta "hibridación" se alcanzan concepciones más profundas y, en la metodología de $A p S$, llegaríamos, sin duda, al punto 6 , ya que supone una implicación y un compromiso frente al destinatario de nuestros productos del proyecto.

Por otra parte, se ha demostrado que la función macro crítica se desarrolla con mayor intensidad a través de la interacción con esta herramienta, como vemos en la investi- 
gación: "Padlet Online Discussion in Enhancing Students' macro Critical Thinking Skills" (Rathakrishnan et al., 2017).

En él, se llevó a cabo un estudio sobre 70 estudiantes del Universiti Utara Malaysia Management Foundation Programme. Se dividió a los alumnos en grupos de cinco y se les asignaron temas de discusión para cada grupo. Las ideas surgidas en los debates internos de cada grupo fueron anotadas en Padlet y compartidas con el resto de la clase. Cada idea debía ser comentada y argumentada por el resto de los alumnos desde diferentes puntos de vista, simulando roles, ofreciendo de este modo, perspectivas críticas razonadas susceptibles de ser refutadas. Las respuestas e interacciones fueron evaluadas, finalmente, por los profesores, utilizando rúbricas de evaluación para pensamiento macro y micro crítico. Algunos de los ítems analizados fueron: la evaluación y el análisis de argumentos, la conexión interdisciplinar, la destreza aclarando problemas, la aportación de soluciones y la matización de generalizaciones (Rathakrishnan et al., 2017: 123-125).

Los resultados fueron concluyentes en varias cuestiones. La primera tiene que ver con la comunicación en sí, mucho más productiva que en intercambios sin Padlet, ya que algunos alumnos no participativos se sienten con mayor libertad en un medio tan dinámico. Creció también significativamente la capacidad de determinar la fiabilidad de las fuentes consultadas. La interacción e interdependencia en la comunicación resultaron factores de enriquecimiento. Padlet les ayudó a analizar las ideas de los demás, establecer vínculos entre ellas y seleccionar la información más relevante, lo que desarrolla, sin duda, la función macrocrítica en el aprendizaje (ibid.).

\section{Conclusiones}

Como hemos señalado con anterioridad, se producen, en la comunidad educativa, numerosos beneficios, tanto individuales como colectivos, por los que la metodología $A p S$ resulta muy positiva. Entre ellos, destacamos:

- Se trata de una "contribución a un cambio cultural que debe promover valores como la solidaridad, la cohesión social y una mayor igualdad" (Martín \& Puig, 2017: 26). Se produce una contribución reflexiva al bien común en un ejercicio de colaboración en varias direcciones: entre compañeros, hacia los colaboradores, entre docentes o cualquiera de los actores que puedan intervenir.

- Implica una conciencia crítica de la realidad social que nos rodea, "Entiende la ciudadanía como participación informada, responsable, activa” (ibid:: 27).

- Pone en marcha, además, competencias esenciales para el futuro laboral de nuestros estudiantes.

El aprendizaje-servicio vincula la mejora de la formación del alumnado con el compromiso cívico. No puede haber un buen aprendizaje profesional sin compromiso cívico, 
pero el compromiso cívico tampoco será de calidad si se desvincula del conocimiento. Estamos ante una metodología que une aprendizaje y valores para dar la máxima fuerza a ambos (Martín \& Puig, 2017: 28).

Entre los objetivos que nos propusimos para el proyecto TradAction en la Universidad de Valladolid, se encuentran los siguientes: desarrollar una formación integral de los alumnos, reforzando aprendizajes vinculados a los valores y los derechos humanos, favorecer entre el alumnado una mayor responsabilidad social, crear un mayor vínculo entre la teoría y la práctica, en entornos reales de aplicación de conceptos y competencias, así como aumentar la motivación de los alumnos en un proyecto que sintieran como "suyo". El $\mathrm{ApS}$ supone la metodología más acertada para alcanzar todos estos objetivos.

Podemos construir un aula más democrática si permitimos intervenir a los alumnos en mayor medida en las decisiones y acciones a llevar a cabo a lo largo del proyecto. Esta colaboración debe pasar por una "presencia" del docente como coordinador y gestor de aprendizajes y comunicaciones. Así, mantendremos el equilibrio entre la presencia socio-cognitiva, la socio-afectiva y la pedagógica, presentes en cualquier aprendizaje (Jézégou, 2014).

La utilización de la herramienta Padlet supone una buena elección para el trabajo colaborativo a este nivel, dada la sencillez de su manejo y lo intuitivo de las funcionalidades que ofrece a los participantes del proyecto. Permite una de las bases del $A p S$ : la cooperación, facilitando intercambios y organización del trabajo. Además, supone un buen instrumento para la evaluación y la coevaluación, permitiendo una reflexión más crítica en el alumno sobre su propio aprendizaje.

Finalmente, la red de aprendizaje creada en el proyecto gracias a las funcionalidades de Padlet, nos permite incluir la metodología global en el marco de "aprendizaje híbrido" (Peraya et al., 2014) en el que se mezclan teoría y práctica, presencialidad y aprendizaje remoto, individual y cooperativo y formal e informal. Se crea, de este modo, un sistema de sinergias capaz de dar respuesta a múltiples perfiles de alumnado.

\section{Referencias bibliográficas}

CAmpo, Laura. 2017. "Evaluar para mejorar los proyectos de Aprendizaje-Servicio en la Universidad” in Rubio, Laura \& Ana Escofet (coord.). Aprendizaje-servicio (ApS): claves para su desarrollo en la Universidad. Barcelona, Octaedro ICE, 125-137.

DeEley, Susan. 2016. El Aprendizaje-Servicio en la educación superior. Teoría, práctica y perspectiva crítica. Madrid, Narcea.

Eyler, J., Giles, D. \& A. Schmiede. 1996. A practitioner's guide to reflection in servicelearning. Nashville, TN., Vanderbilt University.

Goastellec, Gaële. 2014. “Les mutations de l'enseignement supérieur en Europe. Comprendre les transformations à l'œuvre" in La pédagogie universitaire à l'heure du numérique. Questionnement et éclairage de la recherche. Louvain-la-Neuve, De Boeck Supérieur, 55-68. 
Anales de Filología Francesa, n. ${ }^{\circ}$ 29, 2021

AINHOA CUSÁCOVICH

HARASIM, Linda. 1987. "Teaching and learning on-line: Issues in computermediated graduate courses" in Canadian Journal for Educational Communication, 16(2), 117-35.

Harasim, Linda, Hiltz, Roxanne, Teles, Lucio \& Murray Turoff. 2000. Redes de Aprendizaje. Guía para la enseñanza y el aprendizaje en red. Barcelona, Gedisa editorial, Biblioteca de educación.

Hy-Sup (nd). Site du projet Hy-sup. "Dispositifs hybrides, nouvelle perspective pour une pédagogie renouvelée de l'enseignement supérieur": <http://prac-hysup.univ-lyon1.fr/webapp/ website/website.html?id=1578544> [15/01/2021].

JÉzÉGOU, Annie. 2007. "La distance en formation: Cadre opérationnel pour caractériser la distance transactionnelle d'un dispositif' in Actes du congrès AREF 2007. AREF 2007, Strasbourg. http://aref2007.u-strasbg.fr/actes_pdf/AREF2007_Annie_JEZEGOU_337.pdf [15/01/2021].

JÉZÉGou, Annie. 2014. “Chapitre 6. Le modèle de la présence en e-learning. Une modélisation théorique au service de la pratique, notamment en contexte universitaire" in La pédagogie universitaire à l'heure du numérique. Questionnement et éclairage de la recherche. Louvain-la-Neuve, De Boeck Supérieur, 111-120.

Martín, Xus \& Josep M. Puig. 2017. “Aprendizaje-servicio: conceptualización y elementos básicos" in Rubio, Laura \& Ana Escofet (coords.). Aprendizaje- servicio (ApS): claves para su desarrollo en la Universidad. Barcelona, Octaedro ICE, 15-27.

Martínez, Miquel. 2008. Aprendizaje servicio y responsabilidad social de las universidades. Barcelona, Octaedro.

Marton, F., Dall'Alba, G. \& E. Beaty. 1993. "Conceptions of learning” in International Journal of Educational Research, 19(3), 277-300.

Peraya, Daniel, Charlier, Bernadette \& Nathalie Deschryver. 2014. "Une première approche de l'hybridation. Étudier les dispositifs hybrides de formation. Pourquoi? Comment?" in Éducation et formation, mai, $\mathrm{n}^{\circ} \mathrm{e}-301,15-34$.

Peraya, Daniel. 2014. "Distances, absence, proximités et présences: des concepts en déplacement" in Distances et médiations des savoirs, 8 | 2014, mis en ligne le 14 janvier 2015: $<\mathrm{http}$ ://journals.openedition.org/dms/865> [17/01/2021].

Poumay, Marianne. 2014. "Six leviers pour améliorer l'apprentissage des étudiants du supérieur" in Revue internationale de pédagogie de l'enseignement supérieur, 30(1) | 2014, mis en ligne le 07 avril 2014: <http://journals.openedition.org/ripes/778> [08/04/2021].

Rathakrishnan, Mohan, Ahmad, Rahayu \& Lee-Jun Choi. 2017. "Padlet Online Discussion in Enhancing Students' macro Critical Thinking Skills" in International Journal of Latest Research in Humanities and Social Science (IJLRHSS), Vol. 1 - 03, 121-126.

TAPIA, María Nieves. 2017. “Aprendizaje- servicio, un movimiento pedagógico mundial” in Rubio, Laura \& Ana Escofet (coord.). Aprendizaje- servicio (ApS): claves para su desarrollo en la Universidad. Barcelona, Octaedro ICE, 55-68.

VAlluY, Jêrome. 2012. "Vers une pédagogie numérique à l'université? Compte-rendu et discussion de l'ouvrage TIC et métiers de l'enseignement supérieur et de la recherche - Émergences, transformations" in Recueil Alexandries (Col. Recensions): $<$ http://www.reseau-terra.eu/article1234.html> [15/01/2021]. 
